\title{
Management of thyroid nodules
}

\begin{abstract}
Thyroid nodules are common in ENT practice with a prevalence of $4-8 \%$ on clinical palpation and up to $8-76 \%$ when evaluated with an Ultrasound Scan (US). When the nodule size is $1 \mathrm{~cm}$ or more it becomes clinically palpable. Approximately $5 \%$ of thyroid nodules are malignant. Detailed history and clinical examination are important in the evaluation of patients with thyroid nodules. Though US and Fine Needle Aspiration Cytology (FNAC) are useful investigations for thyroid nodules, it may not be possible to arrive at a diagnosis in all cases. Several US features suspicious for malignancy have been identified. Classification systems based on the FNAC results have been devised and are useful in surgical decisionmaking and management. Molecular testing can be used in nodules with FNAC results of atypia or follicular neoplasm, to stratify the risk of cancer. Patients with benign cytology may need a lobectomy for compressive symptoms or cosmetic concerns. Follicular lesions on FNAC need a diagnostic lobectomy followed by completion thyroidectomy, if proven to be malignant. Majority of the Differentiated Thyroid Cancers (DTC) will require total thyroidectomy, radio-active ablation and suppressive thyroxine therapy.
\end{abstract}

Volume 4 Issue I - 2016

Sithamparapillai Ashokkumar,Venkat RS

Leeds General infirmary, UK

Correspondence: Sithamparapillai Ashokkumar, Consultant ENT/Thyroid Surgeon, Leeds General infirmary, 20 Consort Road Kings Lynn PE30 3RL, UK, Tel 07728452210,

Email ashokurmi@hotmail.com

Received: December 0I, 2015 | Published: January 12, 2016

Keywords: thyroid nodules, thyroid carcinoma, fine needle aspiration, mivat, ultrasound scan

Abbreviations: DTC, differentiated thyroid cancers; US, ultrasound scan; BTA, british thyroid association; FNAC, fine needle aspiration cytology; PTC, papillary thyroid cancer; MIVAT, minimally invasive video-assisted thyroidectomy; TAA, transaxillary approach

\section{Introduction}

Thyroid nodules are common clinical findings in ENT practice. Thyroid nodule is a discrete lesion within the thyroid gland. It can present as a solitary nodule or prominent nodule in a patient with Multi Nodular Goitre (MNG). It is found in approximately 4- $8 \%$ of the adult population on clinical palpation and the prevalence is much higher in the range of $8-76 \%$ when the US is used.

A population study in Germany detected thyroid nodules by US in $20 \%$ of the population aged $20-79$ years. Incidence of thyroid nodules is higher in women $(5 \%)$ than men $(1 \%){ }^{1}$ Incidence of thyroid nodules is on the increase due to more and more patients being assessed by the US. Thyroid nodule usually becomes palpable with a diameter of larger than $1 \mathrm{~cm}$. Nevertheless, thyroid cancer is rare. The incidence of thyroid cancer varies from 4 per 100,000 to 13 per 100,000 in the western countries. It is important to note that the incidence of thyroid cancer is on the rise all over the world. This could be due to several factors including, better imaging and histological techniques and diagnosis of micro carcinomas. Thyroid cancer accounts for $1 \%$ of all malignancies and $0.5 \%$ of death from malignancies. ${ }^{1,2}$

Thyroid cancers are the commonest cause of death among all the endocrine malignancies. Only 1 in 20 clinically detected thyroid nodules are malignant. ${ }^{2-11}$ Several risk factors for malignant nodule have been identified (Table 1). Thyroid nodules under the age of 16 are highly likely to be papillary cancers. About $15-30 \%$ of CT PET positive nodules turn out to be malignant. Following are the US features suspicious for malignancy, which would definitely indicate FNAC: cells taller than wide in shape, cell with an irregular spiculated outline with a halo, presence of a hypoechogenicity in a solid nodule, micro calcifications and increased vascularity (Figure 1). Micro calcifications are thought to be due to superimposition of Psammoma bodies upon one another and are most suggestive of Papillary thyroid cancer and have a specificity of up to $95 \%$.

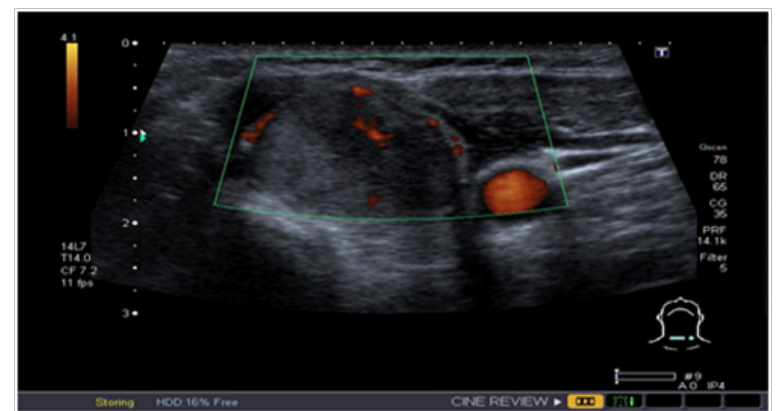

Figure I US showing solid nodule with micro calcification and increased vascularity.

\section{History and examination}

Detailed history and clinical examination of the patient are vital in the management of the patients with thyroid nodules. Incidental detection of thyroid nodule during imaging studies is fairly common in clinical practice but it does not exclude the chance of occurrence of cancer. History should explore the possible risk factors for thyroid cancer as mentioned in Table 1. Rapid increase in size of the lump within a short period is likely to be a sign of malignancy, but if this happens within a very short duration and is associated with pain it may suggest a hemorrhage into a colloid nodule. Exposure to ionizing radiation is a known carcinogenic factor for thyroid cancer. The incidence of malignancy in a previously irradiated thyroid is in the range of 20-50\%. ${ }^{2}$ Progressive hoarseness of voice and stridor should raise the concern for malignancy. Compression symptoms such as dysphagia and breathlessness on exertion should be asked.

Clinical examination of a patient with thyroid nodule should include the assessment of characteristics of the nodule, the presence of central compartment (level VI) or lateral (Level 1 - V) neck nodes and the endoscopic assessment of vocal cord function. Whether the nodule is a solitary one or dominant nodule in a multi nodular goitre may be difficult to establish during palpation, but this can be easily confirmed during US examination. 
Table I Risk factors for thyroid cancer in a patient with a nodule

Gender: Men $>$ Women
Age: $<20$ And $>45$ Yrs
History of Rapid Growth
Past History of Radiation Exposure
Family History Of Thyroid Cancer
Hard on Palpation
Size $>4 \mathrm{Cm}$
CT PET Positive Nodule

\section{Investigations}

\section{Ultra sound scan (US)}

The first line of investigation for a thyroid nodule should be an Ultra Sound scan. High resolution US is highly sensitive to detect nodules as small as $2-4 \mathrm{~mm} .{ }^{11}$ US can not only evaluate the size and character of the nodule but can also assess the presence of neck nodes. If the thyroid gland shows diffuse nodularity or hyperplasia with no defined nodules, FNAC may not be indicated. British Thyroid Association (BTA) has recommended a guideline which differentiates benign and malignant nodules on USS.

\section{Thyroid nodules - ultrasound (U) classification: ( BTA guideline)}

\section{U 1-Normal}

\section{U 2-Benign}

i. Halo. Hyper - / iso-echoic

ii. Cystic changes $+/$ - ring down sign ( colloid)

iii. Micro- cystic / spongiform

iv. Peripheral egg shell calcification.

v. Peripheral vascularity

\section{U 3-Indeterminate / Equivocal}

i. Homogenous, iso-/hyper - echoic, solid, halo ( follicular lesion)

ii. Hypo-echoic, equivocal echogenic foci, cystic changes

iii. Mixed / central vascularity

\section{U 4- Suspicious}

i. Solid, hypo-echoic ( cf thyroid)

ii. Solid, very hypo-echoic ( cf strap muscles)

iii. Lobulated outline

\section{U 5-Malignant}

i. Solid, hypo-echoic, lobulated/ irregular outline, microcalcification (Papillary carcinoma)

ii. Solid, hypo-echoic, lobulated /Irregular outline, globular calcification (Medullary carcinoma)

iii. Intra-nodular vascularity

iv. Shape ( taller $>$ wide)

v. Characteristic associated lymphadenopathy

\section{American thyroid association}

American Thyroid Association has recommended a US based classification for the vast majority of thyroid nodules, into the following categories of ultrasound patterns, which combine several individual sonographic characteristics:-
a. High Suspicion
b. Intermediate suspicion
c. Low suspicion
d. Very low suspicion
e. Benign

\section{Fine needle aspiration cytology (FNAC)}

FNAC is considered as the 'gold standard' in the management of thyroid nodules. ${ }^{1}$ It is a simple, cost-effective, accurate, safe and minimally invasive procedure which can be done in the out-patient setting. FNAC reduces the number of thyroidectomies by $50 \%$, doubles the surgical confirmation of carcinoma and reduces the overall cost of medical care by $25 \% .{ }^{12,13}$ FNAC with Ultrasound guidance increases the diagnostic accuracy, sensitivity and positive predictive value in comparison with palpation guided FNAC. ${ }^{14}$

FNAC is performed under aseptic conditions without local anaesthetic. Verbal consent is obtained and the patient's neck is extended. The nodule is fixed between the index finger and thumb of the non dominant hand. A 23 -gauge needle attached to a $10 \mathrm{ml}$ syringe is advanced slowly into the nodule while applying suction.

The needle is moved back and forth within the nodule to achieve maximum aspiration. The aspirated material is smeared directly on to the glass slides, fixed and sent to the lab immediately. Slides are stained and interpreted by an experienced cytopathologist. The best results depend on the skill of the aspirator and the experience of the cytopathologist. Complications with FNA are rare. Haematoma is the commonly encountered problem. There is no clinical evidence of needle track tumor seeding with FNA. Pneumothorax, thyrotoxicosis, infection induced by FNA and inflammatory reactions have rarely been reported. Based on the cytological findings, FNAC results are classified into various categories with the primary aim of ruling out or confirming malignancy. Two such commonly used systems are given in Table 2.

\section{Tru -cut biopsy (Core biopsy)}

It is usually done under local anaesthetic either by the palpationguided or the US-guided technique. It provides a larger tissue sample which retains its cellular architecture and may enable a more precise histological diagnosis. Tru-cut biopsy of thyroid nodule is useful when repeat FNAC results are inconclusive in a high risk patient (Table 3). It is also useful in suspected lymphoma. This technique is not routinely used due to associated higher risk of haematoma and pain.

\section{Molecular markers}

B-RAF mutation oncogene for papillary thyroid carcinoma (PTC): RAF mutation oncogene stands for Rapidly Acclerated Fibrosarcoma. This oncogene codes for RAF kinases which enchances fibrosarcoma induction. There are three different RAF kinase family members: A-RAF, B-RAF and C-RAF. B-RAF V600E mutation is almost exclusive to (Papillary) Thyroid cancer. V600E mutation denotes substitution of Valline (V) to glutamic acid (E) at position 600 . The molecular pathogenesis of the majority of thyroid cancers involve dysregulation of the MAPK and phosphatidylinositol-3 kinase (PI3K)/AKT signaling pathway. ${ }^{12}$ Point mutation in BRAF is found in approximately $45 \%$ of papillary cancers. In nearly all cases 
(98-99\% of cases) activating point mutation of BRAF involves codon 600 and result in V600E mutation. ${ }^{15}$

BRAF V600E mutation analysis can be used in the management of thyroid nodules, especially in patients with indeterminate FNAC such as Thy3a or Thy $3 f$ to exclude cancer. It has high specificity. ${ }^{16}$ Moreover the presence of BRAF V600E mutation in papillary thyroid cancer (PTC) was found to be associated with poor prognostic factors such as extra thyroidal invasion, lymph node metastasis and recurrence (reviewed in Xing 2007). ${ }^{17}$ The overall mortality rate was $5 \%$ in patients with BRAF V600E mutation and only $1 \%$ in BRAF V600E mutation negative patients. ${ }^{12}$ BRAF V600E mutation can also be used in cost-effective ways in patients with Thy4 result on FNAC. Usually, these patients are subjected to diagnostic hemi-thyroidectomy and then to total thyroidectomy if the histology is proven to be malignant. If BRAF V600E mutation is positive on FNA specimen, there is a case for single stage total thyroidectomy.

Table 2 FNAC classification system (UK \& USA) [3]

\begin{tabular}{|c|c|c|}
\hline Description & BTA* ( UK) & $\begin{array}{l}\text { Bethesda } \\
\text { (TBSRTC)* USA }\end{array}$ \\
\hline Non Diagnostic for Cytological Diagnosis & $\begin{array}{l}\text { Thy I } \\
\text { Cystic Lesion (Thylc) }\end{array}$ & I Cystic Fluid only \\
\hline Non- Neoplastic / Benign & $\begin{array}{l}\text { Thy } 2 \\
\text { Thy 2c } \\
\text { Colloid Cyst }\end{array}$ & II \\
\hline Neoplasm Possible / Atypia of Undetermined Significance & Thy $3 a$ & $\begin{array}{l}\text { III or Follicular Lesion of Undetermined } \\
\text { Significance }\end{array}$ \\
\hline Neoplasm Possible or Suggesting Follicular Neoplasm & Thy $3 f$ & $\begin{array}{l}\text { IV - Follicular Neoplasm or Suspicious Follicular Neoplasm } \\
\text { or Hurthle Cell (Oncocystic)Type }\end{array}$ \\
\hline Suspicious of Malignancy & Thy 4 & $\mathrm{~V}$ \\
\hline Malignant & Thy 5 & VI \\
\hline
\end{tabular}

*TBSRTC - Bethesda System for Reporting Thyroid Cytopathology.

* BTA - British Thyroid Association

Table 3 Risk stratification and management based on FNAC (based on BTA \& TBSRTC ) [3]

\begin{tabular}{lll}
\hline THY Classification & Risk of Cancer & Follow up and Treatment based on THY Classification \\
\hline $\begin{array}{l}\text { THY I - Non Diagnostic/ Inadequate Sample } \\
\text { THY I c- Non Diagnostic/ Cystic Lesion }\end{array}$ & $0-10 \%$ & Repeat FNAC \\
$\begin{array}{l}\text { THY 2 - Benign Lesion } \\
\text { or }\end{array}$ & $0-3 \%$ & $\begin{array}{l}\text { Low Risk Patients - No Follow Up } \\
\text { THY 2 C - Non Neoplastic Cystic Lesion }\end{array}$ \\
$\begin{array}{l}\text { THY 3a -Atypia, Neoplasm Possible. or THY 3f } \\
\text { - Follicular Neoplasm }\end{array}$ & $5-10 \%$ & THY 3a- Repeat FNAC \\
THY 4 - Suspicious of Malignancy & $20-45 \%$ & THY 3f - Diagnostic Hemi Thyroidectomy \\
Thy 5 - Malignant & $60-70 \%$ & $\begin{array}{l}\text { Needs Diagnostic Hemi-Thyroidectomy } \\
\text { Total Thyroidectomy }\end{array}$ \\
\hline
\end{tabular}

\section{Biochemical evaluation}

Most patients with thyroid nodules also need base line thyroid function test. It is also important to check for thyroid auto-antibodies such as thyroid peroxidase and anti-thyroglobulin antibodies to exclude Hashimoto's thyroiditis and Grave's disease.

\section{CT and MRI scans}

Cross sectional imaging modalities are not indicated in the vast majority of thyroid nodules. CT scan are usually considered in patients with suspected tracheal compression, retro-sternal extension and lung metastasis in cases of malignant nodules. A full body CT scan is indicated in patients with primary or secondary lymphoma of thyroid to stage the disease (Figure 2-4). MRI scans are indicated in cases of thyroid nodules presenting with palpable lateral compartment neck nodes.

\section{Surgical management of thyroid nodules}

Benign cytology: The patients with benign cytology and low risk factors may still need follow up for a further 6-18months considering $5 \%$ false negative FNAC results. ${ }^{1,2}$ US $+/$ - FNAC need to be repeated if nodule grows significantly. Growth of the nodule is considered significant with an increase in diameter of $20 \%$ or more with a minimum increase of $2 \mathrm{~mm} . .^{18}$ A prospective, multi centric, observational study involving 992 patients with thyroid nodule with benign cytology on FNAC over a 5 year follow up showed that nodule growth occurred in $15.4 \%$ of patients, nodules shrank spontaneously in $18.5 \%$ of patients and thyroid cancer developed in $0.3 \%$ of patients. Benign cytology in a patient with high risk factors, as mentioned in Table 1 may need diagnostic lobectomy. Patients with a dominant nodule in a multi nodular goitre with compressive symptoms need total thyroidectomy and post-operative thyroxine replacement therapy. Benign solitary nodules in patients with cosmetic concerns may need lobectomy.

Malignant cytology: Malignant cytology on FNAC needs surgical resection of the primary disease to achieve good loco-regional control in most patients. This also enables a patient with thyroid cancer to undertake post operative radio-iodine (I131) ablation, to treat the microscopic disease.

This article focuses on the investigation and treatment of thyroid nodule and therefore, detailed analysis of treatment of all thyroid cancers is beyond its scope. However, general management guideline 
of differentiated thyroid cancer is outlined below. Differentiated thyroid cancer accounts for $90 \%$ to $95 \%$ of all thyroid cancers and includes the following:-

i. Papillary Thyroid Carcinoma (PTC)

ii. Follicular Thyroid carcinoma (FTC)

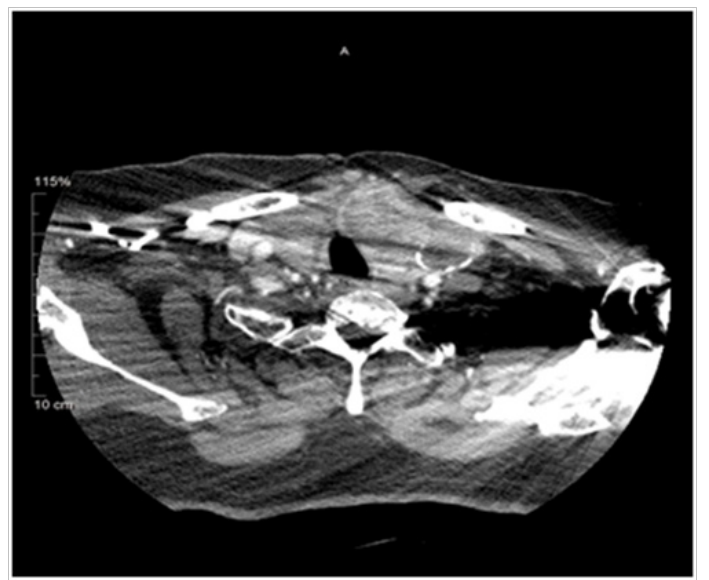

Figure 2 CT Axial view - calcification and tracheal compression.

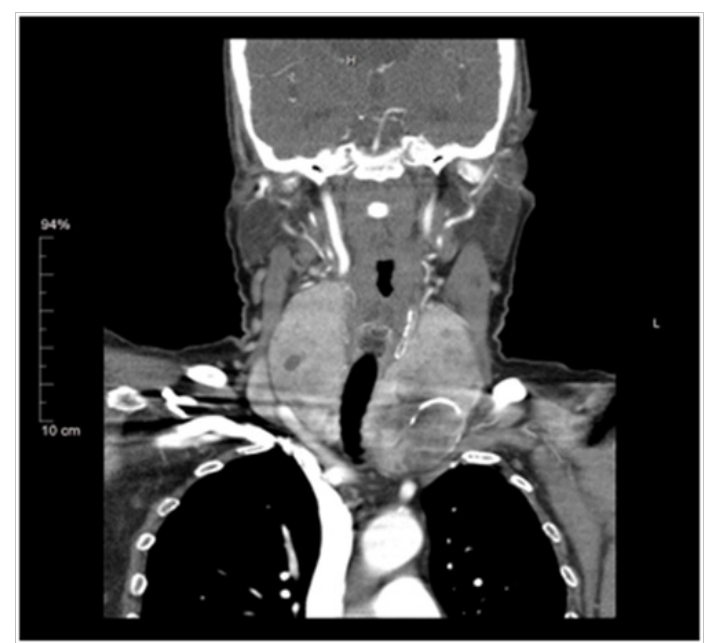

Figure $3 \mathrm{CT}$ Coronal view - tracheal compression.

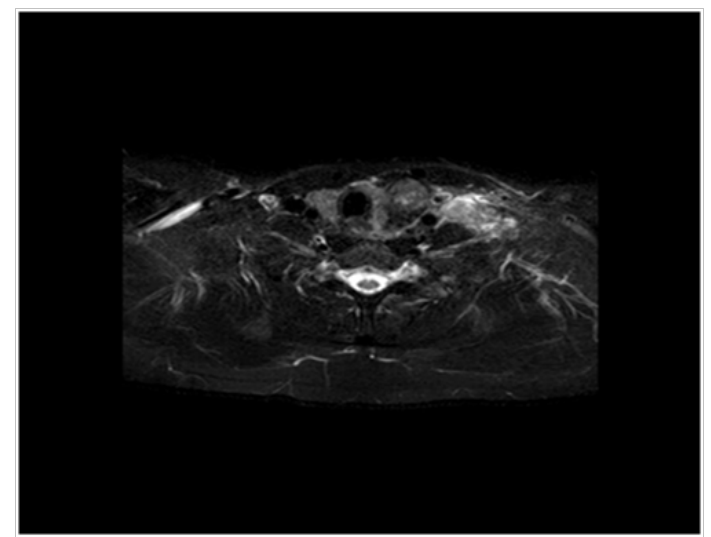

Figure 4 MRI showing left thyroid nodule with lateral neck nodes in papillary cancer.

Papillary thyroid cancer (PTC) is the commonest thyroid cancer accounting for $80 \%$ to $85 \%$ of thyroid cancers. It is associated with good prognosis and the cancers more than $2 \mathrm{~cm}$ are best managed with total thyroidectomy. Routine central compartment dissection is controversial, but it is estimated that $20-50 \%$ of patients have neck node metastasis at the time of diagnosis. ${ }^{2}$

Follicular cancers are diagnosed on histology based on capsular invasion. Thy $3 \mathrm{f}$ and Thy 4 on FNAC need diagnostic lobectomy to confirm follicular cancer. If this is proven on histology, the patient needs completion thyroidectomy later. The role of intra-operative frozen section of thyroid on these patients generates considerable controversy. A study involving 564 patients with thyroid tumors examined the frozen section in $70 \%$ of the patients. Based on this study it was concluded that intra-operative frozen section on thyroid tumors adds very little to surgical management. ${ }^{19}$ Follicular cancers spread haematogenously as compared to PTC which mainly spreads by the lymphatic system. Therefore routine central compartment clearance is not indicated unless there are positive neck nodes on US and FNAC.

For differentiated thyroid cancers (PTC and Follicular cancer), I131 post-operative ablation needs to be undertaken to destroy any residual thyroid cancer tissue, to reduce the chance of any locoregional recurrence and to facilitate long-term surveillance with stimulated thymoglobulin measurements. ${ }^{20}$

\section{Management of incidental nodules}

ENT specialists are encountering an increasing number of incidental thyroid nodules picked up by various imaging modalities such as CT, MRI and CT PET scans. While the incidence of thyroid nodules in the general population is $4-7 \%$, incidence of incidental nodules is approximately $20-30 \%{ }^{21,22}$ Moreover, the malignant rate in incident nodules is higher (7-29\%) as compared to clinically detected nodules $(5 \%){ }^{16}$ Therefore patients with incidental nodules need further investigation with US/FNAC. Further management depends on the FNAC result. A study involving 2000 cases undergoing whole body PET/CT scanning showed incidental increased fluorine-18fluorodeoxyglucose (18 F-FDG) thyroid uptake in 57 patients. Seven of these 57 patients were found to have papillary thyroid cancer on FNAC. If a focal increase in 18 F-FDG uptake by thyroid gland on $\mathrm{PET} / \mathrm{CT}$ is demonstrated, further cytological or histopathological evaluation needs to be undertaken to exclude thyroid cancer. ${ }^{23}$

\section{Thyroid surgery for nodules - Past and present}

When a patient with thyroid nodule needs surgery, it can be performed by different methods depending on nature of the disease, local surgical expertise and the patient choice.
a. Conventional open thyroidectomy
b. Minimal access thyroid surgery
c. Da- Vinci Robotic assisted thyroidectomy

\section{Conventional open thyroidectomy}

Conventional open thyroidectomy was pioneered by Kocher in 1909 and is the most used technique worldwide. ${ }^{20}$ It utilizes a collar neck incision (Kocher incision) ${ }^{24}$ to treat any benign or malignant thyroid problem. This technique inevitably leaves an anterior neck scar. To avoid this cosmetic issue, various other approaches to thyroid surgery have been explored.

\section{Minimally invasive thyroid surgery (MITS)}

The concept of MITS is appealing to the patients as it provides 
excellent cosmetic outcome, reduced hospital stay, minimal postoperative pain, faster recovery and an early return to work. ${ }^{25,26}$ MITS can only be performed on patients who have a small tumor. ${ }^{27}$ MITS can be divided into 3 different techniques: ${ }^{20}$

i. Minimally invasive open surgery (small incision thyroidectomy)

ii. Pure endoscopic techniques

iii. Minimally invasive video-assisted thyroidectomy (MIVAT)

Minimally invasive open surgery is similar to conventional surgery but it utilizes a small incision. Specialized instruments are not needed. In the pure endoscopic method, the anterior neck is approached via the lateral neck, axillary or anterior chest and breast. ${ }^{28,29}$ Surgery is performed with endoscopic assistance with or without carbon dioxide gas insufflation. Minimally invasive video-assisted thyroidectomy (MIVAT) which was first introduced by Miccoli etal in Italy in the late 1990s, is the commonly used MITS technique. A small incision usually $<1.5 \mathrm{~cm}$ is made in the anterior neck skin crease, the operation is performed with video-assisted endoscopes and the specimen is removed via the neck incision.

The disadvantages of MITS are: longer operating time, steep learning curve and added cost of surgery due the use of specialized equipments. $^{30}$ Post-operative complications with MITS are comparable to conventional thyroid surgery. ${ }^{24}$

\section{Da-vinci robotic-assisted thyroidectomy}

Robotic thyroidectomy (RT) using a Da Vinci robotic system is considered in patients who are concerned about the cosmetically visible neck scar associated with conventional thyroidectomy. This technique has been pioneered by Korean surgeons. Selected patients with both benign nodules and differentiated thyroid cancer can be operated by this technique. Patients with a small to average build $(\mathrm{BMI}<30$ ), unilateral follicular lesions (smaller than $5 \mathrm{~cm}$ ) and possessing nodules without thyroiditis are the favorable cases in benign thyroid disease. ${ }^{31}$ For differentiated thyroid cancer a single malignant lesion without nodal or capsular involvement is ideal for a beginner surgeon. ${ }^{28} \mathrm{RT}$ is being used to treat more advanced cancer cases by experienced surgeons in this field.

The most common remote access routes used for RT are the gasless Transaxillary Approach (TAA) and Bilateral Axillo-Breast Approach (BABA). ${ }^{32}$ A $5-6 \mathrm{~cm}$ ipsilateral vertical axillary incision made along the lateral border of the pectoralis major is utilized in the TAA approach. The subcutaneous skin flap is raised from maxilla to the anterior neck and the surgery is performed using the Da Vinci surgical robotic system. As for the BABA approach, two $0.8 \mathrm{~cm}$ sized axillary incisions and two circumareolar incisions measuring $0.8 \mathrm{~cm}$ on the left and $1.2 \mathrm{~cm}$ on the right are used. ${ }^{33}$

The advantages of RT include: an excellent field of view with 3-D imaging, ergonomic movement and an excellent cosmetic outcome. ${ }^{34}$ The main disadvantages of this technique are the advanced training needed and the added cost of the robotic system. RT is as safe and effective as open conventional thyroidectomy for selected patients with benign and malignant thyroid disease with similar complication rates.

\section{Conclusion}

Thyroid nodule is a common ENT problem. Incidental nodules are on the increase due to the use of the several imaging modalities. As 1 in 20 nodules could be malignant, the objectives of the investigations are to characterize the nodule and to rule out cancer. US and FNAC are the key investigations in the management of thyroid nodules. The decision to treat a nodule depends on the type of pathology identified on FNAC and the US characteristics.

\section{Acknowledgments}

None.

\section{Conflicts of interest}

Author declares there are no conflicts of interest.

\section{Funding}

None.

\section{References}

1. Polyzos SA, Kita M, Avramidis A. Thyroid nodules -Stepwise diagnosis and management. Hormones (Athens). 2007;6(2):101-109.

2. Yeung MJ, Serpell JW. Management of the Solitary Thyroid Nodule. Oncologist. 2008;13(2):105-112.

3. Lobo C, McQueen A, Beale T, et al. The UK Royal College of Pathologists Thyroid Fine-Needle Aspiration Diagnostic Classification is a Robust Tool for the clinical management of abnormal Thyroid Nodules. Acta Cytol. 2011;55(6):499-506.

4. Dhyani M, Faquin W, Lubitz CC, et al. How to Interpret Thyroid FineNeedle Aspiration Biopsy Reports: A Guide for the Busy Radiologist in the Era of the Bethesda Classification System. AJR Am J Roentgenol. 2013;201(6):1335-1339

5. Kabaker AS, Tublin ME, Nikiforov YE, et al. Suspicious Ultrasound Characteristics Predict BRAF V600E -positive Papillary Thyroid Cancer. Thyroid. 2012;22(6):585-589.

6. Aspinall SR, Ong SG, Wilson MS, et al. How shall we manage the incidentally found thyroid nodules? Surgeon. 2013;11(2):96-104.

7. Batawil N, Alkordy T. Ultrasonographic features associated with malignancy in cytologically Indterminate thyroid nodules. Eur J Surg Oncol. 2014;40(2):182-186.

8. Choi WJ, Baek JH, Choi YJ, et al. Management of cystic or predominantly cystic thyroid nodules: role of simple aspiration of internal fluid. Endocr Res. 2015;40(4):215-219.

9. Gharib H, Papini E, Paschke R. Thyroid nodules: a review of current guidelines, practices and prospects. Eur $J$ Endocrinol. 2008;159(5):493-505.

10. Huang TW, Lai JH, Wu MY, et al. Systematic review of clinical practice guidelines in the diagnosis and management of thyroid nodules and cancer. BMC Med. 2013;11:191.

11. Anil G, Hegde A, Chong FH. Thyroid nodules: risk stratification for malignancy with ultrasound and guided biopsy. Cancer Imaging. 2011;11(1):209-223.

12. Hsiao SJ, Nikiforov YE. Molecular approaches to thyroid cancer diagnosis. Endocr Relat Cancer. 2014;21(5):T301-T313.

13. Kim SY, Kim EK, Kwak JY, et al. What to do with thyroid nodules showing benign cytology and BRAF (V600E) mutation? A study based on clinical and radiologic features using a highly sensitive analytic method. Surgery. 2015;157(2):354-361.

14. Durante C, Costante G, Lucisano G, et al. The natural history of benign thyroid nodules. Jama. 2015;313(9):926-935.

15. Kazi R, Katna R, Dwivedi RC. Minimal access thyroid surgery- a new dawn? Ann R Coll Surg Eng. 2010;92(5):361-362. 
16. Wilhelm SM, Robinson AV, Krishnamurthy SS,et al. Evaluation and management of incidental thyroid nodules in patients with another primary tumour. Surgery. 2007;142(4):581-586.

17. Iyer NG, Shaha AR, Silver CE, et al. Thyroid incidentomas: to treat or not to treat. Eur Arch Otorhinolaryngol . 2010;267(7):1019-1026.

18. Popoveniuc G, Jonklaas J. Thyroid nodules. Med Clin North Am. 2012;96(2):329-349.

19. Brooks AD, Shaha AR, DuMornay W, et al. Role of fine-needle aspiration biopsy and frozen section analysis in the surgical management of thyroid tumours. Ann Surg Oncol. 2001; 8(2):92-100.

20. Yaylali O, Kirac FS, Yuksel D, et al. Evaluation of focal thyroid lesions incidentally detected in fluorine-18-fluorodeoxyglucose positron emission tomography/computer tomography images. Indian J Cancer. 2014;51(3):236-240.

21. Wilhelm T, Metzig A. Video. Endoscopic minimally invasive thyroidectomy: first clinical experience. Surg Endosc. 2010;24(7):1757-1758.

22. Seybt MW, Terris DJ. Minimally invasive thyroid cancer surgery. Minerva Chir. 2010;65(1):39-43.

23. Dralle H, Machens A, Thanh PN. Minimally invasive compared with conventional thyroidectomy for nodular goiter. Best Pract Res Clin Endocrinol Metab. 2014;28(4):589-599.

24. Billmann F, Bokor-Billmann T, Lapshyn H, et al. Minimal access videoassisted thyroidectomy for benign disease: a retrospective analysis of risk factors for postoperative complications. Int J Surg. 2014; 12(12):1306-1309.

25. Benhidjeb T, Harlaar J, Kerver A, et al. Transoral endoscopic thyroidectomy: part 2: Surgical technique. Chirurg. 2010;81(2):134-138.

26. Lee KE, Kim E, Koo Do H, et al. Robotic thyroidectomy by bilateral axillo-breast approach: review of 1026 cases and surgical completeness. Surg Endosc. 2013;27(8):2955-2562.
27. Lee HY, Yang IS, Hwang SB, et al. Robotic thyroid surgery for papillary thyroid carcinoma: lesson learned from 100 consecutive surgeries. Surg Laparosc Ensoc Percutan Tech. 2015;25(1):27-32.

28. Kang SW, Park JH, Jeong JS, et al. Prospects of robotic thyroidectomy using a gasless, transaxillary approach for the management of thyroid carcinoma. Surg Lapaosc Endosc Percutan Tech. 2011;21(4):223-229.

29. Giannopoulos G, Kang SW, Jeong JJ, et al. Robotic thyroidectomy for benign thyroid disease: a stepwise strategy to the adoption of robotic thyroidectomy( gasless transaxillary approach). Surg Laparosc Endosc Percutan Tech. 2013;23(3):312-315.

30. Lang BH, Wong CK, Tsang JS, et al. A systematic review and metaanalysis comparing outcomes between robotic-assisted thyroidectomy and no-robotic endoscopic thyroidectomy. Journal of surgical Research. 2014; 191(2):389-398

31. Lee S, Lee CR, Lee SC, et al. Surgical completeness of robotic thyroidectomy: a prospective comparision with conventional open thyroidectomy in papillay thyroid carcinoma patients. Surg Endosc. 2014; 28(4):1068-1075.

32. Lee J, Yun JH, Nam KH, et al. Perioperative clinical outcomes after robotic thyroidectomy for thyroid carcinoma: a multicenter study. Surg Endosc. 2011;25(3):906-912.

33. Chai YJ, Lee KE, Youn YK. Can Robotic Thyroidectomy be performed safely in thyroid carcinoma patients? Endocrinol Metab (seoul). 2014;29(3):226-232.

34. Lee KE, Choi JY, Youn YK. Billateral axillo-breast approach robotic thyroidectomy. Surg Laparosc Endosc Percutan Tech. 2011;21(4):230-236.

35. Haugen BR, Alexander EK, Bible KC, et al. American Thyroid Association Management Guidelines for Adult Patients with Thyroid Nodules and Differentiated Thyroid Cancer The American Thyroid Association (ATA) Guidelines Taskforce on Thyroid Nodules and Differentiated Thyroid Cancer. Thyroid 2015 American Thyroid Association. 2015;1-411. 